\title{
Exponential bounds via Stein's method and exchangeable pairs
}

\author{
Patcharee Sumritnorrapong, Kritsana Neammanee, Jiraphan Suntornchost* \\ Department of Mathematics and Computer Science, Faculty of Science, Chulalongkorn University, \\ Bangkok 10330 Thailand
}

*Corresponding author, e-mail: Jiraphan.S@chula.ac.th

Received 21 Jun 2017

Accepted 25 Jun 2018

\begin{abstract}
We apply Stein's method to obtain a non-uniform exponential bound for normal approximations for certain types of random variables. In particular, we establish the bound for a random variable such that its exchangeable pair coupling exists and the distance between the pair is bounded. Using our result, we obtain better bounds for a wide range of applications, such as sums of bounded independent random variables, the combinatorial central limit theorem, and the numbers of descents and inversions of a permutation.
\end{abstract}

KEYWORDS: combinatorial central limit theorem, descent, inversion, non-uniform bound

MSC2010: 60F05

\section{INTRODUCTION}

The central limit theorem and Berry-Esseen theorem play important roles in probability theory and statistics. The theorems guarantee that a certain distribution can be asymptotically approximated by a normal distribution. Several techniques to deal with different variations of the central limit theorem and the Berry-Esseen theorem have been proposed. For example, the Laplace Fourier method can be used to prove the classical central limit theorem, and many significant results related to the limit theorem and Berry-Esseen bounds arise from this method. However, it is quite difficult to apply the method to the case of dependent random variables. The breakthrough finally came from Stein ${ }^{1}$. He introduced an alternative approach to the BerryEsseen normal approximation which is known as Stein's method. His idea is to replace the distance between the characteristic functions in the Fourier analytic method with the distance between the target random variable and the normal distribution. In particular, he showed that $Z \sim \mathscr{N}(0,1)$ if and only if

$$
E\left[Z f(Z)-f^{\prime}(Z)\right]=0,
$$

for any absolutely continuous function $f$ for which this expectation exists.

There are many advantages of Stein's method over the classical Fourier analytic approach. For instance, Stein's method can directly yield the error bound for the normal approximation even when the random variables are not identically distributed or independent. Consequently, Stein's method and its extensions and variations have opened up many applications in distribution approximations such as with the multivariate normal approximation ${ }^{2}$. Reviews of Stein's method and its extensions can be found Refs. 3, 4.

Generally, there are three widely used approaches in Stein's method which are the concentration inequality approach, the inductive approach, and the coupling approach. The coupling approach is one of the most important tools in Stein's method. The key idea of this approach is to construct a new random variable coupling with the target random variable. There are three different couplings we can make which are exchangeable pair ${ }^{5,6}$, zero bias $^{7,8}$ and size bias ${ }^{9,10}$.

The setting of the exchangeable pair coupling is as follows. For a random variable $W$, we say that the pair $\left(W, W^{\prime}\right)$ is an exchangeable pair if for all measurable sets $B$ and $B^{\prime}, P\left(W \in B, W^{\prime} \in B^{\prime}\right)=$ $P\left(W \in B^{\prime}, W^{\prime} \in B\right)$. The exchangeable pair $\left(W, W^{\prime}\right)$ is said to be $\lambda$-Stein pair if ${ }^{6}$

$$
E^{W}\left(W-W^{\prime}\right)=\lambda W,
$$

where $0<\lambda<1$. Generalizations of the $\lambda$-Stein pair construction can be found in Refs. 11-13.

Rinott and Rotar ${ }^{11}$ gave a uniform bound for the error of the normal approximation via the distance 
of exchangeable pair random variables satisfying (1) and $\left|W^{\prime}-W\right| \leqslant A$ for some constant $A$. In particular, for any real number $x$, they found that

$$
\begin{aligned}
& |P(W \leqslant x)-\Phi(x)| \\
& \quad \leqslant \frac{12}{\lambda} \sqrt{\operatorname{Var}\left(E^{W}\left(W^{\prime}-W\right)^{2}\right)}+\frac{48 A^{3}}{\lambda}+\frac{8 A^{2}}{\sqrt{\lambda}} .
\end{aligned}
$$

This result was then used to obtain uniform bounds on the normal approximation in various applications. For example, $W$ can be the numbers of descents and inversions of a permutation ${ }^{14,15}$.

Shao and $\mathrm{Su}^{16}$ established a uniform bound similar to (2) as follows.

$$
\begin{aligned}
& |P(W \leqslant x)-\Phi(x)| \\
& \quad \leqslant \frac{1}{2 \lambda} \sqrt{\operatorname{Var}\left(E^{W}\left(W^{\prime}-W\right)^{2}\right)}+\frac{0.41 A^{3}}{\lambda}+1.5 A .
\end{aligned}
$$

Much effort has been put into obtaining nonuniform bounds for normal approximations in different settings. Chuntee and Neammanee ${ }^{15}$ established non-uniform polynomial bounds for the num-bers of descents and inversions of a permutation, and then improved the non-uniform bounds to non-uniform exponential bounds ${ }^{17}$. In this work, we further improve their technique to obtain a better non-uniform exponential bound for normal approx-imation of certain types of random variables. In particular, we establish the bound for any random variable such that its $\lambda$-Stein pair exists and the distance between the pair is bounded.

The rest of this paper is organized as follows. First, we state our main theorem. Then we apply the theorem to obtain non-uniform exponential bounds for normal approximations in four settings. We provide the proof of the main theorem in the last section.

Theorem 1 Let $W$ be a random variable with zero mean and variance one and $W^{\prime}$ be an exchangeable pair of $W$ such that $E^{W} W^{\prime}=(1-\lambda) W$ with $0<\lambda<$ 1 . Assume that $\delta_{W}\left|W^{\prime}-W\right| \leqslant A$ for some constant $A$. For

$z \in \mathbb{R}$ such that $4 A<|z| \leqslant(0.623 / \lambda) A$,

$$
\begin{array}{r}
P\left(\forall C_{\mathbb{1}}(z)\right) \frac{1}{2 \lambda} \Phi \sqrt{(x) \operatorname{dr}\left(E^{W}\left(W^{\prime}-W\right)^{2}\right)}+C_{2}(z) \frac{0.41 A^{3}}{\lambda} \\
+1.5 A C_{3}(z)
\end{array}
$$

where $\Phi$ is the standard normal distribution and

$$
\begin{aligned}
C_{1}(z)= & \sqrt{\frac{3}{2 \pi} \frac{\mathrm{e}^{-z^{2} / 2}}{|z|}} \\
& +\left(2+\mathrm{e}^{(8 / 3) \lambda^{2}|z|^{3} / A^{3}}\right)^{1 / 2} \mathrm{e}^{-\left(z^{2} / 2\right)\left(\left(\lambda /\left(A^{2}+2 \lambda\right)\right)\right)}, \\
C_{2}(z)= & 0.610\left(\frac{1}{|z|}\left(1+\frac{9 z^{2}}{16}\right) \mathrm{e}^{-(7 / 32) z^{2}}+\frac{\mathrm{e}^{-(1 / 2) z^{2}}}{\sqrt{2 \pi}}\right) \\
& +0.620(1+|z|) \mathrm{e}^{-\lambda z^{2} / 4 A^{2}+\lambda^{2}|z|^{3} / 3 A^{3}} \\
C_{3}(z)= & \frac{8}{3} \mathrm{e}^{2 \lambda|z| / A+4 \lambda^{2}|z|^{3} / 3 A^{3}-\lambda z^{2} / 2 A^{2}}
\end{aligned}
$$

Furthermore, if $\max \left(|z|,|z|^{3}\right) \leqslant 1 / A$, then there exist positive constants $a$ and $C$ such that $C_{i}(z) \leqslant C \mathrm{e}^{-a z^{2}}$ for $i=1,2,3$. Hence

$$
\begin{array}{r}
|P(W \leqslant z)-\Phi(z)| \\
\leqslant C \mathrm{e}^{-a z^{2}\left(\frac{1}{2 \lambda} \sqrt{\operatorname{Var}\left(E^{W}\left(W^{\prime}-W\right)^{2}\right)}\right.} \\
\left.+\frac{0.41 A^{3}}{\lambda}+1.5 A\right) .
\end{array}
$$

The non-uniform bound obtained in Theorem 1 is only applicable to a certain interval of $|z|$. However, in most applications, $A=O(1 / \sqrt{n})$ and $\lambda=$ $O(1 / n)$. Then the lower limit of the interval becomes a constant while the upper limit tends to infinity as $n \rightarrow \infty$. Hence we can guarantee that, for any given large $|z|$, we can find a suitable sample size $n$ such that the theorem is applicable and the bound obtained is still smaller than any existing bound. For the case of small values of $|z|$, the uniform bound (3) is already available ${ }^{16}$.

\section{APPLICATIONS}

In this section, four applications of Theorem 1 are discussed. For each application, we first construct an exchangeable pair $W^{\prime}$ of the target random variable $W$ such that $E^{W} W^{\prime}=(1-\lambda) W$ with $0<\lambda<1$ and $\delta_{W}\left|W^{\prime}-W\right| \leqslant A$ for some constant $A$ and then compute a bound for the term $\operatorname{Var}\left(E^{W}\left(W-W^{\prime}\right)^{2}\right)$.

\section{Independent bounded random variables}

Let $X_{1}, X_{2}, \ldots, X_{n}$ be independent and not necessarily identically distributed random variables with zero means and finite variances. Define $W=$ $\sum_{i=1}^{n} X_{i}$ and assume that $\operatorname{Var}(W)=1$.

Chen and Shao ${ }^{18}$ gave a uniform bound for a normal approximation for the sum of independent random variables with bounded condition $\left|X_{i}\right| \leqslant \delta$ for $i=1,2, \ldots, n$ :

$$
\sup _{z \in \mathbb{R}}|P(W \leqslant z)-\Phi(z)| \leqslant 3.3 \delta .
$$


Chaidee ${ }^{19}$ gave a non-uniform exponential bound improving this bound for large $z$ as follows. For every real number $z,|P(W \leqslant z)-\Phi(z)| \leqslant C_{\delta} \mathrm{e}^{-|z| / 2} \delta$, where $C_{\delta}=4.45+2.21 \mathrm{e}^{2 \delta+\left(\delta^{-2}\left(\mathrm{e}^{2 \delta}-1-2 \delta\right)\right)}$.

Here we show that the application of our main theorem to the sum of independent bounded random variables can improve the non-uniform exponential bound in Ref. 19. The theorem is stated below.

Theorem 2 Assume that $\left|X_{i}\right| \leqslant \delta$ for $i=1,2, \ldots, n$. Then for fixed $z \in \mathbb{R}$ such that $\max \left(|z|,|z|^{3}\right) \leqslant 1 / 2 \delta$ with $8 \delta<|z| \leqslant 1.256 n \delta$,

$$
\begin{aligned}
& |P(W \leqslant z)-\Phi(z)| \leqslant \sqrt{\frac{3}{8 \pi}} \sqrt{n} \delta^{2} \frac{\mathrm{e}^{-z^{2} / 2}}{|z|} \\
& +\frac{\sqrt{n} \delta^{2}}{2}\left(2+\mathrm{e}^{1 / 6 n^{2} \delta^{4}}\right)^{1 / 2} \mathrm{e}^{-z^{2} /\left(8 n \delta^{2}+4\right)} \\
& +2.001 n \delta^{3} \mathrm{e}^{-7 z^{2} / 32}\left(\frac{1}{|z|}+\frac{9|z|}{16}\right)+\frac{2.001 n \delta^{3}}{\sqrt{2 \pi}} \mathrm{e}^{-z^{2} / 2} \\
& +2.034 n \delta^{3}(1+|z|) \mathrm{e}^{-z^{2} / 16 n \delta^{2}+1 / 48 n^{2} \delta^{4}} \\
& +8 \delta \mathrm{e}^{-z^{2} / 8 n \delta^{2}+1 / 2 n \delta^{2}+1 / 12 n^{2} \delta^{4}} .
\end{aligned}
$$

Furthermore, if $\delta=K / \sqrt{n}$ where $K \geqslant 0.548$, then for $8 K<|z| \leqslant n^{1 / 6} /(2 K)^{1 / 3}$,

$$
\begin{aligned}
|P(W \leqslant z)-\Phi(z)| & \leqslant \frac{1}{\sqrt{n}} \mathrm{e}^{-3 z^{2} / 64 K^{2}}\left(10.243 K^{4}\right. \\
& \left.+1.879 K^{3}+1.524 K^{2}+0.719 K\right) .
\end{aligned}
$$

Proof: Let $X_{i}^{\prime}$ be an independent copy of $X_{i}$ and let $I$ be a uniformly distributed random variable on $\{1,2, \ldots, n\}$ which is independent of $\left\{X_{i}, X_{i}^{\prime}, i=\right.$ $1,2, \ldots, n\}$. Define $W^{\prime}=W-X_{I}+X_{I}^{\prime}$. Then $\left(W, W^{\prime}\right)$ is an exchangeable pair ${ }^{3}$ satisfying (1) with $\lambda=$ $1 / n$ and $\delta_{W} \leqslant 2 \delta$. From Ref. 18, $E^{W}\left(W-W^{\prime}\right)^{2}=$ $(1 / n)\left(1+\sum_{i=1}^{n} E^{W}\left(X_{i}^{2}\right)\right)$. Hence

$$
\operatorname{Var}\left(E^{W}\left(W-W^{\prime}\right)^{2}\right) \leqslant \frac{\delta^{4}}{n}
$$

Substituting $A=2 \delta, \lambda=1 / n$, and (4) into the bound in Theorem 1, we obtain, for $8 \delta<|z| \leqslant$ $1.256 n \delta$,

$$
\begin{aligned}
C_{1}(z)= & \sqrt{\frac{3}{2 \pi} \frac{\mathrm{e}^{-z^{2} / 2}}{z}} \\
& +\left(2+\mathrm{e}^{(1 / 3)|z|^{3} / n^{2} \delta^{3}}\right)^{1 / 2} \mathrm{e}^{-z^{2} /\left(8 n \delta^{2}+4\right)}, \\
C_{2}(z)= & 0.610\left(\frac{1}{|z|}\left(1+\frac{9 z^{2}}{16}\right) \mathrm{e}^{-7 z^{2} / 32}+\frac{\mathrm{e}^{-z^{2} / 2}}{\sqrt{2 \pi}}\right) \\
& +0.620(1+|z|) \mathrm{e}^{-z^{2} / 4 n \delta^{2}+|z|^{3} / 24 n^{2} \delta^{3}}, \\
C_{3}(z)= & \frac{8}{3} \mathrm{e}^{|z| / n \delta+|z|^{3} / 6 n^{2} \delta^{3}-z^{2} / 8 n \delta^{2}} .
\end{aligned}
$$

Hence

$$
\begin{aligned}
& \mid P(W \leqslantz)-\Phi(z) \mid \leqslant \sqrt{\frac{3}{8 \pi}} \sqrt{n} \delta^{2} \frac{\mathrm{e}^{-z^{2} / 2}}{|z|} \\
&+ \frac{\sqrt{n} \delta^{2}}{2}\left(2+\mathrm{e}^{1 / 6 n^{2} \delta^{4}}\right)^{1 / 2} \mathrm{e}^{-z^{2} /\left(8 n \delta^{2}+4\right)} \\
&+2.001 n \delta^{3} \mathrm{e}^{-7 z^{2} / 32}\left(\frac{1}{|z|}+\frac{9|z|}{16}\right)+\frac{2.001 n \delta^{3}}{\sqrt{2 \pi}} \mathrm{e}^{-z^{2} / 2} \\
&+2.034 n \delta^{3}(1+|z|) \mathrm{e}^{-z^{2} / 16 n \delta^{2}+1 / 48 n^{2} \delta^{4}} \\
&+8 \delta \mathrm{e}^{-z^{2} / 8 n \delta^{2}+1 / 2 n \delta^{2}+1 / 12 n^{2} \delta^{4}}
\end{aligned}
$$

where we use the fact that $\max \left(|z|,|z|^{3}\right) \leqslant 1 / 2 \delta$ and direct calculation to obtain the inequality. Furthermore, if $\delta=K / \sqrt{n}$, where $K \geqslant 0.548$, then for $8 K<|z| \leqslant n^{1 / 6} /(2 K)^{1 / 3}$,

$$
\begin{aligned}
|P(W \leqslant z)-\Phi(z)| & \leqslant \mathrm{e}^{-3 z^{2} / 64 K^{2}} \frac{K^{2}}{\sqrt{n}}\left(\sqrt{\frac{3}{8 \pi}}+\frac{1}{2}\left(2+\mathrm{e}^{1 / 6 K^{4}}\right)^{1 / 2}\right) \\
+ & \mathrm{e}^{-3 z^{2} / 64 K^{2}} \frac{2.001 K^{3}}{\sqrt{n}}\left(\frac{1+36 K^{2}}{8 K} \mathrm{e}^{3-14 K^{2}}+\frac{1}{\sqrt{2 \pi}}\right) \\
& +\mathrm{e}^{-3 z^{2} / 64 K^{2}} \frac{2.034 K^{3}}{\sqrt{n}}(1+8 K) \mathrm{e}^{1 / 48 K^{4}-1} \\
& +\frac{8 K}{\sqrt{n}} \mathrm{e}^{-3 z^{2} / 64 K^{2}+1 / 2 K^{2}+1 / 12 K^{4}-5 z^{2} / 64 K^{2}} \\
\leqslant & \frac{1}{\sqrt{n}} \mathrm{e}^{-3 z^{2} / 64 K^{2}}\left(10.243 K^{4}+1.879 K^{3}\right. \\
& \left.+1.524 K^{2}+0.719 K\right),
\end{aligned}
$$

where we use the fact that $\mathrm{e}^{-z^{2} /\left(8 K^{2}+4\right)} \leqslant$ $\mathrm{e}^{-3 z^{2} / 64 K^{2}}$ for $K \geqslant 0.548$ and the fact that $\mathrm{e}^{3 z^{2} / 64 K^{2}-7 z^{2} / 32}(1 /|z|+9|z| / 16)$ and $(1+|z|) \mathrm{e}^{-z^{2} / 64 K^{2}}$ are decreasing in $z$ on $[8 K, \infty)$ to obtain $(5)$.

\section{Combinatorial central limit theorem}

For $n \geqslant 2$, let $a_{i j}$ be an $n \times n$ matrix of real numbers and $\pi$ be a random permutation of $\{1,2, \ldots, n\}$ and

$$
\mu=n a_{. .}, \quad \sigma^{2}=\frac{1}{n-1} \sum_{i, j=1}^{n}\left(a_{i j}-a_{i .}-a_{. j}+a_{. .}\right)^{2}
$$

where

$$
a_{i .}=\frac{1}{n} \sum_{j=1}^{n} a_{i j}, \quad a_{\cdot j}=\frac{1}{n} \sum_{i=1}^{n} a_{i j}, \quad a_{. .}=\frac{1}{n^{2}} \sum_{i, j=1}^{n} a_{i j} .
$$

Define $W=\sum_{i=1}^{n} x_{i \pi(i)}$, where $x_{i j}=\left(a_{i j}-a_{i .}-\right.$ $\left.a_{. j}+a_{. .}\right) / \sigma$. Then $E(W)=0, \operatorname{Var}(W)=1$ and $W$ is approximately standard normal distributed. The 
asymptotic normality for $W$ under various conditions were shown in Refs. 20,21. Bahr ${ }^{22}$ and Ho and $\mathrm{Chen}^{23}$ obtained a uniform bound for $W$ with the rate $O(1 / \sqrt{n})$ under boundedness conditions $\sup _{i, j}\left|x_{i j}\right|=O(1 / \sqrt{n})$. Ho and Chen's result is that, for every real number $z$,

$$
\left|P\left(W_{n} \leqslant z\right)-\Phi(z)\right| \leqslant C \beta,
$$

where $\beta=(1 / n) \sum_{i, j=1}^{n}\left|x_{i j}\right|^{3}$. Bolthausen ${ }^{24}$ gave a uniform bound for $W$ with finite third moment conditions. His result is as follows. There exists an absolute constant $C>0$, such that

$$
\sup _{z \in \mathbb{R}}|P(W \leqslant z)-\Phi(z)| \leqslant C \beta
$$

which has an $O(1 / \sqrt{n})$ rate of convergence. Chen and Fang ${ }^{25}$ calculated the constant of the uniform bound for $W$ in (6) to be 451, and obtained a bound in the form of

$$
\sup _{z \in \mathbb{R}}|P(W \leqslant z)-\Phi(z)| \leqslant 451 \delta
$$

for the bounded condition $\sup _{i, j}\left|x_{i j}\right| \leqslant \delta$. Chaidee ${ }^{19}$ gave a non-uniform bound for the normal approximation for $W$ with the same bounded condition where $\delta=O(1 / \sqrt{n})$. The bound is, for any real number $z$,

$$
|P(W \leqslant z)-\Phi(z)| \leqslant \frac{C \delta}{\left(1+|z|^{3}\right)},
$$

where $C$ is a positive constant.

We now apply our main theorem to improve the non-uniform polynomial bound to an exponential bound in the form of $\mathrm{e}^{-c z^{2}}$. The obtained bound is much smaller than the polynomial bound given in (7) for sufficiently large $z$.

Theorem 3 Assume that $\sup _{i, j}\left|x_{i j}\right| \leqslant \delta$. Then for fixed $z \in \mathbb{R}$ such that $\max \left(|z|,|z|^{3}\right) \leqslant 1 / 4 \delta$ with $16 \delta<|z| \leqslant 1.256(n-1) \delta$,

$$
\begin{aligned}
&|P(W \leqslant z)-\Phi(z)| \leqslant \sqrt{\frac{33}{4 \pi}} \sqrt{n} \delta^{2} \frac{\mathrm{e}^{-z^{2} / 2}}{|z|} \\
&+ \sqrt{\frac{11}{2}} \sqrt{n} \delta^{2}\left(2+\mathrm{e}^{1 / 6 n^{2} \delta^{4}}\right)^{1 / 2} \mathrm{e}^{-z^{2} /\left(16 n \delta^{2}+8\right)} \\
&+8.004 n \delta^{3} \mathrm{e}^{-7 z^{2} / 32}\left(\frac{1}{|z|}+\frac{9|z|}{16}\right)+\frac{8.004 n \delta^{3}}{\sqrt{2 \pi}} \mathrm{e}^{-z^{2} / 2} \\
&+8.135 n \delta^{3}(1+|z|) \mathrm{e}^{-z^{2} / 32 n \delta^{2}+1 / 48 n^{2} \delta^{4}} \\
&+16 \delta \mathrm{e}^{-z^{2} / 16 n \delta^{2}+1 / 2 n \delta^{2}+1 / 12 n^{2} \delta^{4}} .
\end{aligned}
$$

Furthermore, if $\delta=K / \sqrt{n}$ where $K \geqslant 0.548$, then for $16 K<|z| \leqslant n^{1 / 6} /(4 K)^{1 / 3}$,

$$
\begin{aligned}
|P(W \leqslant z)-\Phi(z)| & \leqslant \frac{1}{\sqrt{n}} \mathrm{e}^{-3 z^{2} / 128 K^{2}}\left(22.194 K^{4}\right. \\
& \left.+4.582 K^{3}+6.961 K^{2}+0.012 K\right)
\end{aligned}
$$

Proof: For a random permutation $\pi$ on the symmetric group $S_{n}$, let a pair $(I, J)$ be a random variable independent of $\pi$ with $P(I=i$ and $J=j)=1 / n(n-$ 1) for every $i, j \in\{1,2, \ldots, n\}$ such that $i \neq j$. Define $\pi^{\prime}$ and $W^{\prime}$ by

$$
\pi^{\prime}(i)= \begin{cases}\pi(i), & i \notin\{I, J\} \\ \pi(J), & i=I, \\ \pi(I), & i=J,\end{cases}
$$

and $W^{\prime}=W^{\prime}(\pi)=W\left(\pi^{\prime}\right)=\sum_{i=1}^{n} x_{i \pi^{\prime}(i)}$. From Ref. $6,\left(\pi, \pi^{\prime}\right)$ is an exchangeable pair. Hence $\left(W, W^{\prime}\right)$ is also an exchangeable pair. Since $W^{\prime}-$ $W=x_{I \pi(J)}+x_{J \pi(I)}-x_{I \pi(I)}-x_{J \pi(J)}, \sup _{i, j}\left|x_{i j}\right| \leqslant \delta$, and $\delta_{W} \leqslant 4 \delta$. Furthermore, the exchangeable pair $\left(W, W^{\prime}\right)$ satisfies (1) with $\lambda=2 /(n-1)$. Following the proof in Ref. 6, we can show that

$$
\begin{aligned}
& \operatorname{Var}\left(E^{W}\left(W^{\prime}-W\right)^{2}\right) \leqslant \operatorname{Var}\left(E^{\pi}\left(W^{\prime}-W\right)^{2}\right) \\
& \leqslant \frac{4}{n^{2}(n-1)^{2}}\left[\sum_{i, j=1}^{n} x_{i j}^{4}+n \sum_{i, j=1}^{n} x_{i j}^{4}\right. \\
& \left.\quad+\sum_{i, j=1}^{n} \operatorname{Var}\left(x_{i \pi(j)} x_{j \pi(i)}+x_{i \pi(i)} x_{j \pi(j)}\right)\right] .
\end{aligned}
$$

Applying the inequality $(a+b)^{2} \leqslant 2\left(a^{2}+b^{2}\right)$ and the Hölder inequality, we can show that

$$
\sum_{i, j=1}^{n} \operatorname{Var}\left(x_{i \pi(j)} x_{j \pi(i)}+x_{i \pi(i)} x_{j \pi(j)}\right) \leqslant 8 \sum_{i, j=1}^{n} x_{i j}^{4} .
$$

Hence

$$
\operatorname{Var}\left(E^{W}\left(W^{\prime}-W\right)^{2}\right) \leqslant \frac{88 \delta^{4}}{n} .
$$

Substituting (8), $A=4 \delta$, and $\lambda=2 /(n-1)$ into the bound in Theorem 1 we obtain, for $16 \delta<z \leqslant$ $1.256(n-1) \delta$,

$$
\begin{aligned}
& C_{1}(z) \leqslant \sqrt{\frac{3}{2 \pi}} \frac{\mathrm{e}^{-z^{2} / 2}}{|z|} \\
& +\left(2+\mathrm{e}^{(2 / 3)|z|^{3} / n^{2} \delta^{3}}\right)^{1 / 2} \mathrm{e}^{-z^{2} /\left(16 n \delta^{2}+8\right)} \\
& C_{2}(z) \leqslant 0.610\left(\frac{1}{|z|}\left(1+\frac{9 z^{2}}{16}\right) \mathrm{e}^{-7 z^{2} / 32}+\frac{\mathrm{e}^{-z^{2} / 2}}{\sqrt{2 \pi}}\right) \\
& +0.620(1+|z|) \mathrm{e}^{-z^{2} / 32 n \delta^{2}+|z|^{3} / 12 n^{2} \delta^{3}} \\
& C_{3}(z) \leqslant \frac{8}{3} \mathrm{e}^{2|z| / n \delta+|z|^{3} / 3 n^{2} \delta^{3}-z^{2} / 16 n \delta^{2}} \text {. }
\end{aligned}
$$


Hence

$$
\begin{aligned}
& |P(W \leqslant z)-\Phi(z)| \leqslant \sqrt{\frac{33}{4 \pi}} \sqrt{n} \delta^{2} \frac{\mathrm{e}^{-z^{2} / 2}}{|z|} \\
& +\sqrt{\frac{11}{2}} \sqrt{n} \delta^{2}\left(2+\mathrm{e}^{1 / 6 n^{2} \delta^{4}}\right)^{1 / 2} \mathrm{e}^{-z^{2} /\left(16 n \delta^{2}+8\right)} \\
& +8.004 n \delta^{3} \mathrm{e}^{-7 z^{2} / 32}\left(\frac{1}{|z|}+\frac{9|z|}{16}\right)+\frac{8.004 n \delta^{3}}{\sqrt{2 \pi}} \mathrm{e}^{-z^{2} / 2} \\
& +8.135 n \delta^{3}(1+|z|) \mathrm{e}^{-z^{2} / 32 n \delta^{2}+1 / 48 n^{2} \delta^{4}} \\
& +16 \delta \mathrm{e}^{-z^{2} / 16 n \delta^{2}+1 / 2 n \delta^{2}+1 / 12 n^{2} \delta^{4}},
\end{aligned}
$$

where we use the fact that $\max \left(|z|,|z|^{3}\right) \leqslant 1 / 4 \delta$ and a direct calculation to obtain the inequality. Furthermore, if $\delta=K / \sqrt{n}$ where $K \geqslant 0.548$, then for $16 K<|z| \leqslant n^{1 / 6} /(4 K)^{1 / 3}$,

$$
\begin{aligned}
\mid P( & \leqslant z)-\Phi(z) \mid \\
\leqslant & \mathrm{e}^{-3 z^{2} / 128 K^{2}} \frac{K^{2}}{\sqrt{n}}\left(\sqrt{\frac{33}{4 \pi}}+\sqrt{\frac{11}{2}}\left(2+\mathrm{e}^{2 / 6 K^{4}}\right)^{1 / 2}\right) \\
+ & \mathrm{e}^{-3 z^{2} / 128 K^{2}} \frac{8.004 K^{3}}{\sqrt{n}}\left(\frac{1+144 K^{2}}{16 K} \mathrm{e}^{6-56 K^{2}}+\frac{1}{\sqrt{2 \pi}}\right) \\
& +\mathrm{e}^{-3 z^{2} / 128 K^{2}} \frac{8.135 K^{3}}{\sqrt{n}}(1+16 K) \mathrm{e}^{-2+1 / 48 K^{2}} \\
& +\frac{16 K}{\sqrt{n}} \mathrm{e}^{-3 z^{2} / 128 K^{2}+1 / 2 K^{2}+1 / 12 K^{4}-5 z^{2} / 128 K^{2}} \\
\leqslant & \frac{1}{\sqrt{n}} \mathrm{e}^{-3 z^{2} / 128 K^{2}}\left(22.194 K^{4}+4.582 K^{3}\right. \\
& \left.+6.961 K^{2}+0.012 K\right),
\end{aligned}
$$

where we use the fact that $\mathrm{e}^{-z^{2} /\left(16 K^{2}+8\right)} \leqslant$ $\mathrm{e}^{-3 z^{2} / 128 K^{2}}$ for $K \geqslant 0.548$ and the fact that $\mathrm{e}^{3 z^{2} / 128 K^{2}-7 z^{2} / 32}(1 /|z|+9|z| / 16)$ and $(1+|z|) \mathrm{e}^{-z^{2} / 128 K^{2}}$ are decreasing in $z$ on $[16 K, \infty)$ to obtain (9).

\section{The numbers of descents and inversions of a permutation}

For a random permutation $\pi$ on the symmetric group $S_{n}$, let $\operatorname{Des}(\pi)$ be the number of descents of permutation $\pi$. Then $\operatorname{Des}(\pi)$ is the number of pairs $(i, i+1)$ with $1 \leqslant i \leqslant n-1$ such that $\pi(i)>\pi(i+1)$. Similarly, let $\operatorname{Inv}(\pi)$ be the number of inversions of permutation $\pi$. Then $\operatorname{Inv}(\pi)$ is the number of pairs $(i, j)$ with $1 \leqslant i<j \leqslant n$ such that $\pi(i)>\pi(j)$. The expectations and variances of the numbers of descents and inversions are $E(\operatorname{Des}(\pi))=\frac{1}{2}(n-1)$, $\operatorname{Var}(\operatorname{Des}(\pi))=\frac{1}{12}(n+1), E(\operatorname{Inv}(\pi))=\frac{1}{4} n(n-1)$ and $\operatorname{Var}(\operatorname{Inv}(\pi))=\frac{1}{72} n(n-1)(2 n+5)$, respectively ${ }^{14}$. The asymptotic normality for the number of descents is proved in Ref. 26 and for the number of inversions is proved in Ref. 27.
Fulman ${ }^{14}$ gave uniform bounds of the normal approximation to the numbers of descents and inversions. The exchangeable pair techniques of Stein's method and the theorem in Ref. 11 were used to prove the results ${ }^{14}$

$$
\begin{aligned}
& \sup _{z \in \mathbb{R}} \mid P\left(\frac{\operatorname{Des}(\pi)-E(\operatorname{Des}(\pi))}{\sqrt{\operatorname{Var}(\operatorname{Des}(\pi))} \leqslant z)-\Phi(z) \mid \leqslant \frac{C_{1}}{\sqrt{n}},}\right. \\
& \sup _{z \in \mathbb{R}}\left|P\left(\frac{\operatorname{Inv}(\pi)-E(\operatorname{Inv}(\pi))}{\sqrt{\operatorname{Var}(\operatorname{Inv}(\pi))}} \leqslant z\right)-\Phi(z)\right| \leqslant \frac{C_{2}}{\sqrt{n}},
\end{aligned}
$$

where $C_{1}$ and $C_{2}$ are positive constants.

Chuntee and Neammanee ${ }^{15}$ calculated the constants in Fulman's results which are 1096 and 5421, respectively. In their work, they also proposed a method to reduce the constants to be 13.42 and 14.24 for the number of descents and the number of inversions, respectively. Furthermore, they generalized the two bounds to be non-uniform bounds in polynomial form. Chuntee and Neammanee ${ }^{17}$ improved the rate of convergences for descents and inversions from the polynomial bounds to exponential bounds. Their results are as follows. For sufficiently large $n$,

$$
\begin{aligned}
\mid P\left(\frac{\operatorname{Des}(\pi)-E(\operatorname{Des}(\pi))}{\sqrt{\operatorname{Var}(\operatorname{Des}(\pi))}} \leqslant z\right) & -\Phi(z) \mid \\
& \leqslant \frac{51.25}{\sqrt{n}} \mathrm{e}^{-|z| / 4}
\end{aligned}
$$

and

$$
\begin{aligned}
\mid P\left(\frac{\operatorname{Inv}(\pi)-E(\operatorname{Inv}(\pi))}{\sqrt{\operatorname{Var}(\operatorname{Inv}(\pi))}} \leqslant z\right) & -\Phi(z) \mid \\
& \leqslant \frac{792.71}{\sqrt{n}} \mathrm{e}^{-|z| / 4} .
\end{aligned}
$$

Here we provide smaller exponential bounds for normal approximations to the numbers of descents and inversions.

Theorem 4 For fixed $z \in \mathbb{R}$ and $n>z^{6}$. If $|z|>8 \sqrt{3}$, then

$$
\begin{aligned}
\mid P\left(\frac{\operatorname{Des}(\pi)-E(\operatorname{Des}(\pi))}{\sqrt{\operatorname{Var}(\operatorname{Des}(\pi))}}\right. & \leqslant z)-\Phi(z) \mid \\
& \leqslant \frac{10.980}{\sqrt{n}} \mathrm{e}^{-z^{2} / 32} .
\end{aligned}
$$


Table 1 Comparisons of the constant $C$ for the number of descents and inversions in the form of $C / \sqrt{n}$.

\begin{tabular}{lccc}
\hline bound & $z=25$ & $z=100$ & $z=1000$ \\
\hline$(10)$ & 0.3454 & $7.2 \times 10^{-10}$ & $1.4 \times 10^{-107}$ \\
$(12)$ & $2.0 \times 10^{-7}$ & $2.2 \times 10^{-135}$ & $2.2 \times 10^{-13571}$ \\
$(11)$ & 1.5303 & $1.2 \times 10^{-8}$ & $2.2 \times 10^{-106}$ \\
$(13)$ & 0.1391 & $5.4 \times 10^{-44}$ & $1.2 \times 10^{-4522}$ \\
\hline
\end{tabular}

Theorem 5 For fixed $z \in \mathbb{R}$ and $n>z^{6}$, if $|z|>24$, then

$$
\begin{aligned}
\left|P\left(\frac{\operatorname{Inv}(\pi)-E(\operatorname{Inv}(\pi))}{\sqrt{\operatorname{Var}(\operatorname{Inv}(\pi))}} \leqslant z\right)-\Phi(z)\right| \\
\leqslant \frac{93.467}{\sqrt{n}} \mathrm{e}^{-z^{2} / 96} .
\end{aligned}
$$

Remark 1 For sufficiently large $n$, the bounds given in Theorems 4 and 5 are smaller than the ones given in Ref. 17 when $z>8 \sqrt{3}$ and $z>24$ for the cases of descents and inversions, respectively.

From Table 1, we can see that our bounds (12, 13) are respectively smaller than the exponential bounds $(10,11)$ in Ref. 17 when $n$ satisfies the condition that $n>z^{6}$. The improvements are clearly seen for large values of $z$.

Proof of Theorem 4: For $\pi \in S_{n}$, define

$$
W:=\frac{\operatorname{Des}(\pi)-E(\operatorname{Des}(\pi))}{\sqrt{\operatorname{Var}(\operatorname{Des}(\pi))}},
$$

and

$$
\pi^{\prime}(i)= \begin{cases}\pi(i), & i \notin\{I, I+1, \ldots, n\}, \\ \pi(i+1), & i \in\{I, I+1, \ldots, n-1\}, \\ \pi(I), & i=n,\end{cases}
$$

where $I$ is a uniform random variable taking values on $\{1,2, \ldots, n\}$. The permutation $\pi^{\prime}$ was shown by Fulman ${ }^{14}$ to be an exchangeable pair of $\pi$. Consequently,

$$
W^{\prime}:=\frac{\operatorname{Des}\left(\pi^{\prime}\right)-E\left(\operatorname{Des}\left(\pi^{\prime}\right)\right)}{\sqrt{\operatorname{Var}\left(\operatorname{Des}\left(\pi^{\prime}\right)\right)}}
$$

is an exchangeable pair of $W$. Moreover, the exchangeable pair $\left(W, W^{\prime}\right)$ satisfies (1) with $\lambda=2 / n$ and $\delta_{W} \leqslant 2 \sqrt{3} / \sqrt{n}$.

Chuntee and Neammanee ${ }^{15}$ showed that

$$
\operatorname{Var}\left(E^{W}\left(W^{\prime}-W\right)^{2}\right) \leqslant \frac{25.6}{n^{3}} .
$$

Substituting (15), $A=2 \sqrt{3} / \sqrt{n}$ and $\lambda=2 / n$ into Theorem 1 , we have, for $|z|>8 \sqrt{3}$,

$$
\begin{aligned}
C_{1}(z)= & \sqrt{\frac{3}{2 \pi}} \frac{\mathrm{e}^{-z^{2} / 2}}{|z|}+\left(2+\mathrm{e}^{(4 / 9 \sqrt{3})|z|^{3} / \sqrt{n}}\right)^{1 / 2} \mathrm{e}^{-z^{2} / 16} \\
C_{2}(z)= & 0.610\left(\frac{1}{|z|}\left(1+\frac{9 z^{2}}{16}\right) \mathrm{e}^{-(7 / 32) z^{2}}+\frac{\mathrm{e}^{-z^{2} / 2}}{\sqrt{2 \pi}}\right) \\
& +0.620(1+|z|) \mathrm{e}^{-z^{2} / 24+(1 / 18 \sqrt{3})|z|^{3} / \sqrt{n}} \\
C_{3}(z)= & \frac{8}{3} \mathrm{e}^{(2 / \sqrt{3})|z| / \sqrt{n}+(2 / 9 \sqrt{3})|z|^{3} / \sqrt{n}-z^{2} / 12} .
\end{aligned}
$$

Hence

$$
\begin{aligned}
& |P(W \leqslant z)-\Phi(z)| \\
& \leqslant \frac{\sqrt{25.6}}{4 \sqrt{n}} \mathrm{e}^{-z^{2} / 32}\left[\sqrt{\frac{3}{2 \pi}} \frac{\mathrm{e}^{-15 z^{2} / 32}}{|z|}\right. \\
& \left.\quad+\sqrt{2+\mathrm{e}^{4 / 9 \sqrt{3}}} \mathrm{e}^{-z^{2} / 32}\right] \\
& +\frac{\mathrm{e}^{-z^{2} / 32}}{\sqrt{n}}\left[5.199\left(\frac{16+9 z^{2}}{16|z|} \mathrm{e}^{-(6 / 32) z^{2}}+\frac{\mathrm{e}^{-(15 / 32) z^{2}}}{\sqrt{2 \pi}}\right)\right. \\
& \left.+5.284(1+|z|) \mathrm{e}^{-z^{2} / 96+1 / 18 \sqrt{3}}\right] \\
& \quad+\mathrm{e}^{-z^{2} / 32} \frac{8 \sqrt{3}}{\sqrt{n}} \mathrm{e}^{2 / \sqrt{3}+2 / 9 \sqrt{3}-5 z^{2} / 96} \\
& \leqslant \frac{10.980}{\sqrt{n}} \mathrm{e}^{-z^{2} / 32},
\end{aligned}
$$

where we use the fact that $n>z^{6}$ to obtain (16) and $\sqrt{3 / 2 \pi} \mathrm{e}^{-15 z^{2} / 32} /|z|+$ $\left(2+\mathrm{e}^{4 / 9 \sqrt{3}}\right)^{1 / 2} \mathrm{e}^{-z^{2} / 32} \leqslant 0.0045, \quad((16+$ $\left.\left.9 z^{2}\right) / 16|z|\right) \mathrm{e}^{-6 z^{2} / 32}+\left(\mathrm{e}^{-(15 / 32) z^{2}} / \sqrt{2 \pi}\right) \leqslant$ $1.83 \times 10^{-15}, \quad(1+|z|) \mathrm{e}^{-z^{2} / 96+1 / 18 \sqrt{3}} \leqslant 2.0762$, $\mathrm{e}^{2 / \sqrt{3}+2 / 9 \sqrt{3}-(5 / 96) z^{2}} \leqslant 0.0002$, for $|z|>8 \sqrt{3}$ to obtain the last inequality.

Proof of Theorem 5: Similarly, for

$$
W:=\frac{\operatorname{Inv}(\pi)-E(\operatorname{Inv}(\pi))}{\sqrt{\operatorname{Var}(\operatorname{Inv}(\pi))}}
$$

define the exchangeable pair random variable $W^{\prime}$ by

$$
W^{\prime}:=\frac{\operatorname{Inv}\left(\pi^{\prime}\right)-E\left(\operatorname{Inv}\left(\pi^{\prime}\right)\right)}{\sqrt{\operatorname{Var}\left(\operatorname{Inv}\left(\pi^{\prime}\right)\right)}} .
$$

Fulman $^{14}$ showed that the exchangeable pair $\left(W, W^{\prime}\right)$ satisfies (1) with $\lambda=2 / n$ and $\delta_{W} \leqslant 6 / \sqrt{n}$. Hence $^{15}$

$$
\operatorname{Var}\left(E^{W}\left(W^{\prime}-W\right)^{2}\right) \leqslant \frac{30.8}{n^{3}} .
$$


Substituting (17), $A=6 / \sqrt{n}$, and $\lambda=2 / n$ into Theorem 1, we obtain, for $|z|>24$,

$$
\begin{aligned}
C_{1}(z)= & \sqrt{\frac{3}{2 \pi}} \frac{\mathrm{e}^{-z^{2} / 2}}{|z|}+\left(2+\mathrm{e}^{(4 / 81)|z|^{3} / \sqrt{n}}\right)^{1 / 2} \mathrm{e}^{-z^{2} / 20} \\
C_{2}(z)= & 0.610\left(\frac{1}{|z|}\left(1+\frac{9 z^{2}}{16}\right) \mathrm{e}^{-(7 / 32) z^{2}}+\frac{\mathrm{e}^{-z^{2} / 2}}{\sqrt{2 \pi}}\right) \\
& +0.620(1+|z|) \mathrm{e}^{-z^{2} / 72+|z|^{3} / 162 \sqrt{n}} \\
C_{3}(z)= & \frac{8}{3} \mathrm{e}^{(2 / 3)|z| / \sqrt{n}+(2 / 81)|z|^{3} / \sqrt{n}-z^{2} / 36} .
\end{aligned}
$$

Hence

$$
\begin{aligned}
& |P(W \leqslant z)-\Phi(z)| \\
& \leqslant \frac{\sqrt{30.8}}{4 \sqrt{n}} \mathrm{e}^{-z^{2} / 96}\left[\sqrt{\frac{3}{2 \pi}} \frac{\mathrm{e}^{-47 z^{2} / 96}}{|z|}\right. \\
& \left.+\left(2+\mathrm{e}^{4 / 81}\right)^{1 / 2} \mathrm{e}^{-19 z^{2} / 480}\right] \\
& \quad+\frac{1}{\sqrt{n}} \mathrm{e}^{-z^{2} / 96}\left[2 7 . 0 1 1 \left(\frac{16+9 z^{2}}{16|z|} \mathrm{e}^{-(5 / 24) z^{2}}\right.\right. \\
& \left.\left.+\frac{\mathrm{e}^{-(47 / 96) z^{2}}}{\sqrt{2 \pi}}\right)+27.454(1+|z|) \mathrm{e}^{-z^{2} / 288+1 / 162}\right] \\
& \quad+\mathrm{e}^{-z^{2} / 96} \frac{24}{\sqrt{n}} \mathrm{e}^{2 / 3+2 / 81-5 z^{2} / 288} \\
& \leqslant \frac{93.467}{\sqrt{n}} \mathrm{e}^{-z^{2} / 96},
\end{aligned}
$$

where we use the fact that $n>z^{6}$ to obtain (18) and $\sqrt{3 / 2 \pi} \mathrm{e}^{-47 z^{2} / 96 /|z|+}$ $\left(2+\mathrm{e}^{4 / 81}\right)^{1 / 2} \mathrm{e}^{-19 z^{2} / 480} \leqslant 2.19 \times 10^{-10}$, $\left(\left(16+9 z^{2}\right) / 16|z|\right) \mathrm{e}^{-5 z^{2} / 24}+\left(\mathrm{e}^{-47 z^{2} / 96} / \sqrt{2 \pi}\right) \leqslant$ $1.04 \times 10^{-51}, \quad(1+|z|) \mathrm{e}^{-z^{2} / 288+1 / 162} \leqslant 3.4044$, $\mathrm{e}^{2 / 3+2 / 81-5 z^{2} / 288} \leqslant 9.07 \times 10^{-5}$, for $|z|>24$ to obtain the last inequality.

\section{PROOF OF MAIN RESULT}

Recall that the exchangeable pair $\left(W, W^{\prime}\right)$ satisfies $E^{W}\left(W-W^{\prime}\right)=\lambda W$ and $\delta_{W} \leqslant A$. Chen and Shao ${ }^{18}$ proved that

$$
E W g(W)=E \int_{-\infty}^{\infty} g^{\prime}(W+t) \hat{K}(t) \mathrm{d} t,
$$

where

$$
\begin{aligned}
\hat{K}(t)=\frac{1}{2 \lambda}\left(W^{\prime}-W\right)[\mathbb{I}(0 \leqslant t & \left.\leqslant W^{\prime}-W\right) \\
& \left.-\mathbb{I}\left(W^{\prime}-W \leqslant t \leqslant 0\right)\right],
\end{aligned}
$$

and $g$ is a continuous and piecewise continuously differentiable function. We can see that $\hat{K}(t)$ is always non-negative and $\hat{K}(t)=0$ for $|t|>\delta_{W}$ and

$$
\int_{-\infty}^{\infty} \hat{K}(t) \mathrm{d} t=\frac{1}{2 \lambda}\left(W^{\prime}-W\right)^{2} \leqslant \frac{A^{2}}{2 \lambda} .
$$

To prove the main result, we first obtain an exponential bound for the moment of $W$.

Lemma 1 Let $s>0$ and $s A \leqslant 1.256$. Then

$$
E \mathrm{e}^{s W} \leqslant \mathrm{e}^{A^{2} s^{2} / 4 \lambda+A^{3} s^{3} / 3 \lambda} \text {. }
$$

Proof: We first prove the inequality $\mathrm{e}^{x}-1 \leqslant 2 x$ for $0 \leqslant x \leqslant 1.256$. Note that $\mathrm{e}^{x}-1=2 x$ when $x=$ 0 . Let $g(x)=\left(\mathrm{e}^{x}-1\right) / 2 x$, for $x>0$. Then $g$ is an increasing function on $(0, \infty)$ and $g(1.256)=$ 0.9997. This implies that $g(x) \leqslant 1$ for $0<x$ $\leqslant 1.256$. Hence $\mathrm{e}^{x}-1 \leqslant 2 x$ for $0 \leqslant x \leqslant 1.256$.

For $s \in(0, \infty)$, let $f: \mathbb{R} \rightarrow \mathbb{R}$ be defined by $f(w)$ $=\mathrm{e}^{s w}$ and $h_{W}:(0, \infty) \rightarrow \mathbb{R}$ be defined by $h_{W}(t)=E$ $\mathrm{e}^{t W}$. Following Ref. 17, we can show that

$$
h_{W}^{\prime}(s) \leqslant \frac{s A^{2}}{2 \lambda} E \mathrm{e}^{s W}+\frac{A^{3} s^{2}}{\lambda} E \mathrm{e}^{s W},
$$

where we use the fact that $0 \leqslant s \delta_{W} \leqslant s A \leqslant 1.256$, the property of exchangeable pair (20), and the inequality $\mathrm{e}^{x}-1 \leqslant 2 x$ for $0 \leqslant x \leqslant 1.256$ to obtain the inequality. Dividing the inequality by $h_{W}(s)$ and then integrating we obtain

$$
h_{W}(s) \leqslant \mathrm{e}^{s^{2} A^{2} / 4 \lambda+A^{3} s^{3} / 3 \lambda} .
$$

Proof of Theorem 1: By the symmetry of the standard normal distribution, $\Phi(z)=1-\Phi(-z)$, it suffices to prove the main result for the case $4 A<$ $z \leqslant 0.623 A / \lambda$. Stein's equation for the standard normal approximation is

$$
g^{\prime}(w)-w g(w)=\mathbb{I}(w \leqslant z)-\Phi(z),
$$

where $\Phi$ is the standard normal distribution, $g$ : $\mathbb{R} \rightarrow \mathbb{R}$ is a continuous and piecewise differentiable function. The solution $g_{z}$ of Stein's equation (21) is given by ${ }^{6}$

$$
g_{z}(w)= \begin{cases}\sqrt{2 \pi} \mathrm{e}^{w^{2} / 2} \Phi(w)[1-\Phi(z)], & w \leqslant z \\ \sqrt{2 \pi} \mathrm{e}^{w^{2} / 2} \Phi(z)[1-\Phi(w)], & w>z\end{cases}
$$

and

$$
\begin{aligned}
& g_{z}^{\prime}(w) \\
& \quad= \begin{cases}(1-\Phi(z))\left(1+\sqrt{2 \pi} w \mathrm{e}^{w^{2} / 2} \Phi(w)\right), & w<z, \\
\Phi(z)\left(-1+\sqrt{2 \pi} w \mathrm{e}^{w^{2} / 2}(1-\Phi(w))\right), & w>z,\end{cases}
\end{aligned}
$$


satisfying the following properties: for all $s, t, w \in \mathbb{R}$,

$$
\begin{aligned}
& 0<g_{z}(w) \leqslant \min \left(\frac{\sqrt{2 \pi}}{4}, \frac{1}{|z|}\right), \quad \text { for } z \neq 0, \\
&\left|g_{z}^{\prime}(w)\right| \leqslant 1,\left|g_{z}^{\prime}(s)-g_{z}^{\prime}(t)\right| \leqslant 1,28 \\
&\left|g_{z}^{\prime}(w+s)-g_{z}^{\prime}(w+t)-\int_{t}^{s} h(w+u) \mathrm{d} u\right| \\
& \leqslant \mathbb{I}(z-\max (s, t)<w<z-\min (s, t)) .
\end{aligned}
$$

For $\varepsilon>0$, using (22) and the fact that

$$
\begin{aligned}
& 1-\Phi(z) \leqslant \frac{e^{-z^{2} / 2}}{\sqrt{2 \pi} z} \text { for } z>0, \\
& \Phi(z) \leqslant \frac{\mathrm{e}^{-z^{2} / 2}}{\sqrt{2 \pi}|z|} \quad \text { for } z<0
\end{aligned}
$$

we have

$$
\begin{aligned}
0 \leqslant & g_{z}^{\prime}(w)=(1-\Phi(z))\left(1+\sqrt{2 \pi} w \mathrm{e}^{w^{2} / 2} \Phi(w)\right) \\
\leqslant & \frac{\mathrm{e}^{-z^{2} / 2}}{\sqrt{2 \pi} z}, \quad \text { for } w \leqslant 0 \\
& \left|g_{z}^{\prime}(w)\right|<1, \quad \text { for } w>\frac{z}{1+\varepsilon}
\end{aligned}
$$

and

$$
\begin{aligned}
0 & <g_{z}^{\prime}(w) \\
& \leqslant(1-\Phi(z))\left(1+\sqrt{2 \pi}\left(\frac{z}{1+\varepsilon}\right) \mathrm{e}^{z^{2} / 2(1+\varepsilon)^{2}}\right) \\
& \leqslant \frac{\mathrm{e}^{-z^{2} / 2}}{\sqrt{2 \pi} z}+\mathrm{e}^{\left(z^{2} / 2\right)\left(1 /(1+\varepsilon)^{2}-1\right)}, \quad \text { for } 0<w \leqslant \frac{z}{1+\varepsilon} .
\end{aligned}
$$

By using the inequality $(a+b)^{2} \leqslant 2\left(a^{2}+b^{2}\right)$ for $a, b \in$ $\mathbb{R}$, we obtain

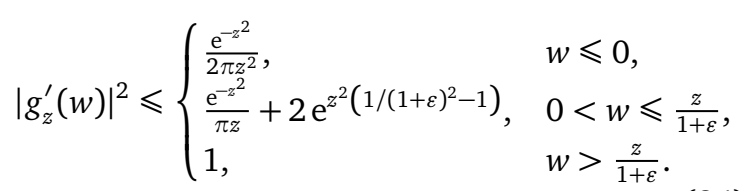

Following Ref. 17,

$$
|P(W \leqslant z)-\Phi(z)| \leqslant\left|T_{1}\right|+\left|T_{2}\right|,
$$

where

$$
\begin{aligned}
& T_{1}=E g_{z}^{\prime}(W)-E g_{z}^{\prime}(W) \int_{-\infty}^{\infty} \hat{K}(t) \mathrm{d} t \\
& T_{2}=E \int_{-\infty}^{\infty}\left(g_{z}^{\prime}(W)-g_{z}^{\prime}(W+t)\right) \hat{K}(t) \mathrm{d} t .
\end{aligned}
$$

To bound $\left|T_{1}\right|$, we follow the technique used in Ref. 17 to obtain

$$
\left|T_{1}\right| \leqslant \sqrt{E\left(g_{z}^{\prime}(W)\right)^{2}} \sqrt{E\left(1-\frac{1}{2 \lambda} E^{W}\left(W^{\prime}-W\right)^{2}\right)^{2}} .
$$

By using (1), we obtain

$$
E\left(W-W^{\prime}\right)^{2}=E\left(E^{W}\left(W-W^{\prime}\right)^{2}\right)=2 \lambda
$$

and therefore

$$
E\left(1-\frac{1}{2 \lambda} E^{W}\left(W^{\prime}-W\right)^{2}\right)^{2}=\frac{\operatorname{Var}\left(E^{W}\left(W^{\prime}-W\right)^{2}\right)}{4 \lambda^{2}} .
$$

To give a bound for $E\left|g_{z}^{\prime}(W)\right|^{2}$, we apply the properties of Stein's solution $g_{z}$ stated in (24). Hence

$$
\begin{gathered}
E\left|g_{z}^{\prime}(W)\right|^{2}=E\left|g_{z}^{\prime}(W)\right|^{2} \mathbb{I}(W \leqslant 0) \\
+E\left|g_{z}^{\prime}(W)\right|^{2} \mathbb{I}\left(0<W \leqslant \frac{z}{1+\varepsilon}\right) \\
+E\left|g_{z}^{\prime}(W)\right|^{2} \mathbb{I}\left(W>\frac{z}{1+\varepsilon}\right) \\
\leqslant \frac{\mathrm{e}^{-z^{2}}}{2 \pi z^{2}} P(W \leqslant 0) \\
+\left(\frac{\mathrm{e}^{-z^{2}}}{\pi z^{2}}+2 \mathrm{e}^{z^{2}\left(1 /(1+\varepsilon)^{2}-1\right)}\right) P\left(0<W \leqslant \frac{z}{1+\varepsilon}\right) \\
+P\left(W>\frac{z}{1+\varepsilon}\right) .
\end{gathered}
$$

Applying Markov's inequality and Lemma 1, we obtain

$$
\begin{aligned}
P\left(W>\frac{z}{1+\varepsilon}\right) & =P\left(\frac{2 \lambda z W}{A^{2}}>\frac{2 \lambda z^{2}}{A^{2}(1+\varepsilon)}\right) \\
& \leqslant \mathrm{e}^{\left(-2 \lambda z^{2}\right) / A^{2}(1+\varepsilon)} E \mathrm{e}^{2 \lambda z W / A^{2}} \\
& \leqslant \mathrm{e}^{\left(-2 \lambda z^{2}\right) / A^{2}(1+\varepsilon)+\lambda z^{2} / A^{2}+8 \lambda^{2} z^{3} / 3 A^{3}}
\end{aligned}
$$

Choosing $\varepsilon$ such that $1 /(1+\varepsilon)=(-\lambda+$ $\left.\sqrt{A^{4}+A^{2} \lambda+\lambda^{2}}\right) / A^{2}$ gives

$$
\begin{aligned}
& E\left|g_{z}^{\prime}(W)\right|^{2} \leqslant \frac{3 \mathrm{e}^{-z^{2}}}{2 \pi z^{2}} \\
& +\left(2+\mathrm{e}^{(8 / 3) \lambda^{2} z^{3} / A^{3}}\right) \mathrm{e}^{-z^{2}\left(\lambda / A^{2}\right)\left(\left(-2 \lambda+2 \sqrt{A^{4}+A^{2} \lambda+\lambda^{2}}\right) / A^{2}-1\right)} \\
& \quad \leqslant \frac{3 \mathrm{e}^{-z^{2}}}{2 \pi z^{2}}+\left(2+\mathrm{e}^{(8 / 3) \lambda^{2} z^{3} / A^{3}}\right) \mathrm{e}^{-z^{2}\left(\left(\lambda /\left(A^{2}+2 \lambda\right)\right)\right)} .
\end{aligned}
$$


Combining (26)-(28),

$$
\begin{aligned}
& \left|T_{1}\right| \\
& \leqslant\left[\frac{3 \mathrm{e}^{-z^{2}}}{2 \pi z^{2}}+\left(2+\mathrm{e}^{8 \lambda^{2} z^{3} / 3 A^{3}}\right) \mathrm{e}^{-z^{2} \lambda /\left(A^{2}+2 \lambda\right)}\right]^{1 / 2} \\
& \times \frac{1}{2 \lambda} \sqrt{\operatorname{Var}\left(E^{W}\left(W^{\prime}-W\right)^{2}\right)} \\
& \leqslant\left[\sqrt{\frac{3}{2 \pi}} \frac{\mathrm{e}^{-z^{2} / 2}}{z}\right. \\
& \left.+\left(2+\mathrm{e}^{8 \lambda^{2} z^{3} / 3 A^{3}}\right)^{1 / 2} \mathrm{e}^{-z^{2} \lambda / 2\left(A^{2}+2 \lambda\right)}\right] \\
& \times \frac{1}{2 \lambda} \sqrt{\operatorname{Var}\left(E^{W}\left(W^{\prime}-W\right)^{2}\right)} .
\end{aligned}
$$

To bound $\left|T_{2}\right|$, we notice that

$$
\begin{aligned}
\left|T_{2}\right| & \leqslant E \int_{-\infty}^{\infty}\left|g_{z}^{\prime}(W)-g_{z}^{\prime}(W+t)\right| \hat{K}(t) \mathrm{d} t \\
& \leqslant T_{21}+T_{22}+T_{23},
\end{aligned}
$$

where

$$
\begin{aligned}
& T_{21}=E \mathbb{I}\left(\delta_{W} \geqslant \frac{z}{4}\right) \int_{-\infty}^{\infty}\left|g_{z}^{\prime}(W)-g_{z}^{\prime}(W+t)\right| \hat{K}(t) \mathrm{d} t \\
& T_{22}=E \mathbb{I}\left(\delta_{W}<\frac{z}{4}\right) \\
& \quad \times \int_{-\infty}^{\infty} \mathbb{I}(z-\max (0, t)<W<z-\min (0, t)) \hat{K}(t) \mathrm{d} t \\
& T_{23}=E \mathbb{I}\left(\delta_{W}<\frac{z}{4}\right) \int_{-\infty}^{\infty} \int_{t}^{0} h(W+u) \hat{K}(t) \mathrm{d} u \mathrm{~d} t,
\end{aligned}
$$

and $h(w)=\left(w g_{z}(w)\right)^{\prime}$. The term $T_{21}$ vanishes by the assumption that $z>4 A$. Hence the bound for $T_{2}$ only depends on $T_{22}$ and $T_{23}$. A bound for $T_{22}$ is obtained as follows. For $|t| \leqslant \delta_{W}$ and $z-\delta_{W}<W<$ $z+\delta_{W}$, we have $z-2 A<z-2 \delta_{W}<W+t$ which implies that

$$
\mathrm{e}^{s z(z-2 A)} \leqslant \mathrm{e}^{s z(W+t)} \quad \text { for any } s>0 .
$$

Using (31), we can see that

$$
\begin{aligned}
& \left|T_{22}\right| \\
& \leqslant E \int_{|t| \leqslant \delta_{W}} \mathbb{I}(z-|t|<W<z+|t|) \hat{K}(t) \mathrm{d} t \\
& \leqslant E \int_{|t| \leqslant \delta_{W}} \mathbb{I}\left(z-\delta_{W}<W<z+\delta_{W}\right) \hat{K}(t) \mathrm{d} t \\
& \leqslant \frac{\mathrm{e}^{2 s z A}}{\mathrm{e}^{s z^{2}}} \\
& \quad \times E \int_{|t| \leqslant \delta_{W}} \mathrm{e}^{s z(W+t)} \mathbb{I}\left(z-\delta_{W}<W<z+\delta_{W}\right) \hat{K}(t) \mathrm{d} t .
\end{aligned}
$$

Let $s=\lambda / A^{2}, \delta>0$ and $f_{\delta}: \mathbb{R} \rightarrow \mathbb{R}$ be defined as

$$
f_{\delta}(t)= \begin{cases}0, & t<z-2 \delta, \\ \mathrm{e}^{s z t}(t-z+2 \delta), & z-2 \delta \leqslant t \leqslant z+2 \delta, \\ 4 \delta \mathrm{e}^{s z t}, & t>z+2 \delta .\end{cases}
$$

Then

$$
\begin{gathered}
f_{\delta}^{\prime}(t) \geqslant \begin{cases}\mathrm{e}^{s z t}, & z-2 \delta<t<z+2 \delta, \\
0, & t<z-2 \delta \text { or } t>z+2 \delta,\end{cases} \\
\left|f_{\delta}(t)\right| \leqslant 4 \delta \mathrm{e}^{s z t,} \quad \text { for all } t \in \mathbb{R} .
\end{gathered}
$$

From (19), (33), and (34),

$$
\begin{aligned}
& E \int_{|t| \leqslant \delta_{W}} \mathrm{e}^{s z(W+t)} \mathbb{I}\left(z-\delta_{W}<W<z+\delta_{W}\right) \hat{K}(t) \mathrm{d} t \\
& \quad \leqslant E \int_{|t| \leqslant \delta_{W}}^{\prime} f^{\prime}(W+t) \hat{K}(t) \mathrm{d} t \\
& \quad \leqslant E W f_{\delta_{W}}(W) \leqslant 4 E|W| \delta_{W} \mathrm{e}^{s z W} .
\end{aligned}
$$

From (32) and (35) and using the Hölder inequality and Lemma 1, we obtain

$$
\begin{aligned}
\left|T_{22}\right| & \leqslant \frac{4 A \mathrm{e}^{2 s z A}}{\mathrm{e}^{s z^{2}}}\left(E|W|^{2}\right)^{1 / 2}\left(E \mathrm{e}^{2 s z W}\right)^{1 / 2} \\
& \leqslant \frac{4 A \mathrm{e}^{2 s z A}}{\mathrm{e}^{s z^{2}}}\left(E \mathrm{e}^{2 s z W}\right)^{1 / 2} \\
& \leqslant \frac{4 A \mathrm{e}^{2 s z A}}{\mathrm{e}^{s z^{2}}}\left(\mathrm{e}^{\left(A^{2} / 4 \lambda\right)(2 s z)^{2}+\left(A^{3} / 3 \lambda\right)(2 s z)^{3}}\right)^{1 / 2} \\
& =4 A \mathrm{e}^{2 \lambda z / A+4 \lambda^{2} z^{3} / 3 A^{3}-\lambda z^{2} / 2 A^{2}} .
\end{aligned}
$$

Hence

$$
\left|T_{22}\right| \leqslant 4 A \mathrm{e}^{2 \lambda z / A+4 \lambda^{2} z^{3} / 3 A^{3}-\lambda z^{2} / 2 A^{2}} .
$$

For the bound for $T_{23}$, we notice that

$$
\begin{aligned}
T_{23} & \leqslant E \mathbb{I}\left(\delta_{W}<\frac{z}{4}\right) \\
\times & {\left[\int_{-\infty}^{\infty} \int_{t}^{0} h(W+u) \hat{K}(t) \mathbb{I}\left(W+u \leqslant \frac{3 z}{4}\right) \mathrm{d} u \mathrm{~d} t\right.} \\
& \left.+\int_{-\infty}^{\infty} \int_{t}^{0} h(W+u) \hat{K}(t) \mathbb{I}\left(W+u>\frac{3 z}{4}\right) \mathrm{d} u \mathrm{~d} t\right] .
\end{aligned}
$$

From the definition $h(w)=\left(w g_{z}(w)\right)^{\prime}$ written as

$h(w)$

$$
= \begin{cases}\left(\sqrt{2 \pi}\left(1+w^{2}\right) \mathrm{e}^{w^{2} / 2} \Phi(w)+w\right)[1-\Phi(z)], & w \leqslant z, \\ \left(\sqrt{2 \pi}\left(1+w^{2}\right) \mathrm{e}^{w^{2} / 2}(1-\Phi(w))-w\right) \Phi(z), & w>z,\end{cases}
$$


we can obtain a bound for the function $h$ as follows.

If $w \leqslant 0$, then $h(w) \leqslant 2(1-\Phi(z)) \leqslant 2 \mathrm{e}^{-z^{2} / 2} / \sqrt{2 \pi} z$.

If $0<w \leqslant 3 z / 4$, then

$$
\begin{aligned}
h(w) & \leqslant\left(\sqrt{2 \pi}\left(1+\frac{9 z^{2}}{16}\right) \mathrm{e}^{(9 / 32) z^{2}}+z\right) \frac{\mathrm{e}^{-z^{2} / 2}}{\sqrt{2 \pi} z} \\
& \leqslant \frac{1}{z}\left(1+\frac{9 z^{2}}{16}\right) \mathrm{e}^{-(7 / 32) z^{2}}+\frac{\mathrm{e}^{-z^{2} / 2}}{\sqrt{2 \pi}} .
\end{aligned}
$$

Hence, for $w \leqslant 3 z / 4$,

$$
h(w) \leqslant \frac{1}{z}\left(1+\frac{9 z^{2}}{16}\right) \mathrm{e}^{-(7 / 32) z^{2}}+\frac{\mathrm{e}^{-z^{2} / 2}}{\sqrt{2 \pi}} .
$$

For $w>3 z / 4$, following Ref. 17 we have

$$
h(w) \leqslant 1.001(1+z) \quad \text { for } w>\frac{3 z}{4} .
$$

Using (38) and the fact that

$$
\int_{-\infty}^{\infty} \int_{t}^{0} \hat{K}(t) \mathrm{d} u \mathrm{~d} t \leqslant \frac{1}{4 \lambda}\left|W^{\prime}-W\right|^{3} \leqslant \frac{A^{3}}{4 \lambda},
$$

we obtain

$$
\begin{aligned}
& E \mathbb{I}\left(\delta_{W}<\frac{z}{4}\right) \\
& \quad \times \int_{-\infty}^{\infty} \int_{t}^{0} h(W+u) \hat{K}(t) \mathbb{I}\left(W+u \leqslant \frac{3 z}{4}\right) \mathrm{d} u \mathrm{~d} t \\
& \quad \leqslant \frac{A^{3}}{4 \lambda}\left(\frac{1}{z}\left(1+\frac{9 z^{2}}{16}\right) \mathrm{e}^{-(7 / 32) z^{2}}+\frac{\mathrm{e}^{-z^{2} / 2}}{\sqrt{2 \pi}}\right)
\end{aligned}
$$

Applying Markov's inequality and Lemma 1, we obtain

$P\left(W>\frac{z}{2}\right)=P\left(\frac{\lambda z W}{A^{2}}>\frac{\lambda z^{2}}{2 A^{2}}\right) \leqslant \mathrm{e}^{\left(-\lambda z^{2}\right) / 4 A^{2}+\lambda^{2} z^{3} / 3 A^{3}}$.

Using (39), (40) and (42), we can show that

$$
\begin{aligned}
& E \mathbb{I}\left(\delta_{W}<\frac{z}{4}\right) \\
& \quad \times \int_{-\infty}^{\infty} \int_{t}^{0} h(W+u) \hat{K}(t) \mathbb{I}\left(W+u>\frac{3 z}{4}\right) \mathrm{d} u \mathrm{~d} t \\
& \quad \leqslant 1.001(1+z) E \mathbb{I}\left(\delta_{W}<\frac{z}{4}\right) \\
& \times \int_{-\infty}^{\infty} \int_{t}^{0} \hat{K}(t) \mathbb{I}\left(W+\delta_{W}>\frac{3 z}{4}\right) \mathrm{d} u \mathrm{~d} t \\
& \leqslant 1.001(1+z) E \int_{-\infty}^{\infty} \int_{t}^{0} \hat{K}(t) \mathbb{I}\left(W>\frac{z}{2}\right) \mathrm{d} u \mathrm{~d} t \\
& \quad \leqslant 1.001(1+z) \frac{A^{3}}{4 \lambda} P\left(W>\frac{z}{2}\right) \\
& \leqslant \frac{1.001 A^{3}}{4 \lambda}(1+z) \mathrm{e}^{-\lambda z^{2} / 4 A^{2}+\lambda^{2} z^{3} / 3 A^{3}} .
\end{aligned}
$$

From (37), (41), and (43), we have

$$
\begin{aligned}
\left|T_{23}\right| \leqslant \frac{A^{3}}{4 \lambda} & \left(\frac{1}{z}\left(1+\frac{9 z^{2}}{16}\right) \mathrm{e}^{-(7 / 32) z^{2}}+\frac{\mathrm{e}^{-(1 / 2) z^{2}}}{\sqrt{2 \pi}}\right) \\
& +\frac{1.001 A^{3}}{4 \lambda}(1+z) \mathrm{e}^{-\lambda z^{2} / 4 A^{2}+\lambda^{2} z^{3} / 3 A^{3}} .
\end{aligned}
$$

From (30), (36), and (44),

$$
\begin{aligned}
& \left|T_{2}\right| \leqslant 4 A \mathrm{e}^{2 \lambda z / A+4 \lambda^{2} z^{3} / 3 A^{3}-\lambda z^{2} / 2 A^{2}}+\frac{A^{3}}{4 \lambda} \\
& \times\left(\frac{1}{z}\left(1+\frac{9 z^{2}}{16}\right) \mathrm{e}^{-(7 / 32) z^{2}}+\frac{\mathrm{e}^{-(1 / 2) z^{2}}}{\sqrt{2 \pi}}\right) \\
& +\frac{1.001 A^{3}}{4 \lambda}(1+z) \mathrm{e}^{-\lambda z^{2} / 4 A^{2}+\lambda^{2} z^{3} / 3 A^{3}} .
\end{aligned}
$$

By (25), (29), and (45), we have

$$
\begin{aligned}
& |P(W \leqslant z)-\Phi(z)| \\
& \leqslant\left[\sqrt{\frac{3}{2 \pi}} \frac{\mathrm{e}^{-z^{2} / 2}}{z}\right. \\
& \left.+\left(2+\mathrm{e}^{(8 / 3) \lambda^{2} z^{3} / A^{3}}\right)^{1 / 2} \mathrm{e}^{-\left(z^{2} / 2\right)\left(\left(\lambda /\left(A^{2}+2 \lambda\right)\right)\right)}\right] \\
& \times \frac{1}{2 \lambda} \sqrt{\operatorname{Var}\left(E^{W}\left(W^{\prime}-W\right)^{2}\right)} \\
& +\frac{A^{3}}{4 \lambda}\left(\frac{1}{z}\left(1+\frac{9 z^{2}}{16}\right) \mathrm{e}^{-(7 / 32) z^{2}}+\frac{\mathrm{e}^{-(1 / 2) z^{2}}}{\sqrt{2 \pi}}\right) \\
& +\frac{1.001 A^{3}}{4 \lambda}(1+z) \mathrm{e}^{-\lambda z^{2} / 4 A^{2}+\lambda^{2} z^{3} / 3 A^{3}} \\
& +4 A \mathrm{e}^{2 \lambda z / A+4 \lambda^{2} z^{3} / 3 A^{3}-\lambda z^{2} / 2 A^{2}} \\
& \leqslant C_{1}(z) \frac{1}{2 \lambda} \sqrt{\operatorname{Var}\left(E^{W}\left(W^{\prime}-W\right)^{2}\right)} \\
& +C_{2}(z) \frac{0.41 A^{3}}{\lambda}+1.5 A C_{3}(z)
\end{aligned}
$$

where

$$
\begin{aligned}
C_{1}(z)= & \sqrt{\frac{3}{2 \pi}} \frac{\mathrm{e}^{-z^{2} / 2}}{z} \\
& +\left(2+\mathrm{e}^{(8 / 3) \lambda^{2} z^{3} / A^{3}}\right)^{1 / 2} \mathrm{e}^{-\left(z^{2} / 2\right)\left(\left(\lambda /\left(A^{2}+2 \lambda\right)\right)\right)} \\
C_{2}(z)= & 0.610\left(\frac{1}{z}\left(1+\frac{9 z^{2}}{16}\right) \mathrm{e}^{-(7 / 32) z^{2}}+\frac{\mathrm{e}^{-(1 / 2) z^{2}}}{\sqrt{2 \pi}}\right) \\
& +0.620(1+z) \mathrm{e}^{-\lambda z^{2} / 4 A^{2}+\lambda^{2} z^{3} / 3 A^{3}} \\
C_{3}(z)= & \frac{8}{3} \mathrm{e}^{2 \lambda z / A+4 \lambda^{2} z^{3} / 3 A^{3}-\lambda z^{2} / 2 A^{2}} .
\end{aligned}
$$

Furthermore, if $\max \left(|z|,|z|^{3}\right) \leqslant 1 / A$, then we can see that

$$
\frac{\lambda^{2}|z|^{3}}{A^{3}} \leqslant \frac{\lambda^{2}}{A^{4}}, \quad \frac{\lambda|z|}{A} \leqslant \frac{\lambda}{A^{2}} .
$$

Hence for $i=1,2,3$, there exist positive constants $a$ and $C$ such that $C_{i}(z) \leqslant C \mathrm{e}^{-a z^{2}}$. 
Acknowledgements: The authors thank the referees for their valuable comments. The first author thanks the Development and Promotion of Science and Technology Talents Project (DPST) and the 90th Anniversary of Chulalongkorn University Fund (Ratchadaphiseksomphot Endowment Fund) for financial support.

\section{REFERENCES}

1. Stein C (1972) A bound for the error in the normal approximation to the distribution of a sum of dependent random variables. Proc Sixth Berkeley Symp Math Statist Probab vol 2, 583-602.

2. Goldstein L, Rinott Y (1996) Multivariate normal approximations by Stein's method and size bias couplings. J Appl Probab 33, 1-17.

3. Chen LHY, Goldstein L, Shao QM (2011) Normal approximation by Stein's method, Springer, Heidelberg.

4. Ross N (2011) Fundamentals of Stein's method. Probab Surv 8, 210-93.

5. Röllin A (2008) A note on the exchangeability condition in Stein's method. Stat Probab Lett 78, 1800-6.

6. Stein C (1986) Approximate computation of expectations, Lecture Notes-Monograph Series 7, IMS, Hayward, CA.

7. Goldstein L (2005) Berry-Esseen bounds for combinatorial central limit theorems and pattern occurrences, using zero and size biasing. J Appl Probab 42, 661-83.

8. Goldstein L, Xia A (2006) Zero biasing and a discrete central limit theorem. Ann Probab 34, 1782-806.

9. Arratia R, Baxendale P (2015) Bounded size bias coupling: a Gamma function bound, and universal Dickman-function behavior. Probab Theor Relat Field 162, 411-29.

10. Ghosh S, Goldstein L (2011) Concentration of measures via size-biased couplings. Probab Theor Relat Field 149, 271-8.

11. Rinott Y, Rotar V (1997) On coupling constructions and rates in the CLT for dependent summands with applications to the antivoter model and weighted Ustatistics. Ann Appl Probab 7, 1080-105.

12. Chatterjee $S$ (2007) Stein's method for concentration inequalities. Probab Theor Relat Field 138, 305-21.

13. Reinert G, Röllin A (2009) Multivariate normal approximation with Stein's method of exchangeable pairs under a general linearity condition. Ann Probab 37, 2150-73.

14. Fulman J (2004) Stein's method and non-reversible Markov chains. In: Stein's Method: Expository Lectures and Applications, IMS Lecture Notes Monogr Ser, vol 46, 66-74.

15. Chuntee W, Neammanee K (2015) Bounds on Normal Approximations for the number of Descents and Inversions. Comm Stat Theor Meth 44, 2310-29.

16. Shao QM, Su ZG (2006) The Berry-Esseen bound for character ratios. Proc Am Math Soc 134, 2153-9.
17. Chuntee W, Neammanee K (2017) Exponential bounds for normal approximation of the number of Descents and Inversions. Comm Stat Theor Meth 46, 1218-29.

18. Chen LHY, Shao QM (2005) Stein's method for normal approximation. In: An Introduction to Stein's Method, World Scientific, pp 1-59.

19. Chaidee N (2005) Non-uniform bounds in normal approximation for matrix correlation statistics and independent bounded random variables. PhD's thesis, Department of Mathematics, Faculty of Science, Chulalongkorn Univ.

20. Hoeffding W (1951) A combinatorial central limit theorem. Ann Math Stat 22, 558-66.

21. Motoo M (1957) On the Hoeffding's combinatorial central limit theorem. Ann Inst Stat Math 8, 145-54.

22. Bahr B (1976) Remainder term estimate in a combinatorial limit theorem. $Z$ Wahrscheinlichkeitstheorie Verwandte Gebiete 35, 131-9.

23. Ho ST, Chen LHY (1978) An $L_{p}$ bound for the remainder in a combinatorial central limit theorem. Ann Probab 6, 231-49.

24. Bolthausen E (1984) An estimate of the remainder in a combinatorial central limit theorem. $Z$ Wahrscheinlichkeitstheorie Verwandte Gebiete 66, 379-86.

25. Chen LHY, Fang X (2015) On the error bound in a combinatorial central limit theorem. Bernoulli 21, 335-59.

26. Pitman J (1997) Probabilistic bounds on the coefficients of polynomials with only real zeros. $J$ Combin Theor A 77, 279-303.

27. Bender EA (1973) Central and local limit theorems applied to asymptotic enumeration. $J$ Combin Theor A 15, 91-111.

28. Chen LHY, Shao QM (2001) A non-uniform BerryEsseen bound via Stein's method. Probab Theor Relat Field 120, 236-54. 